\title{
The poetry of space
}

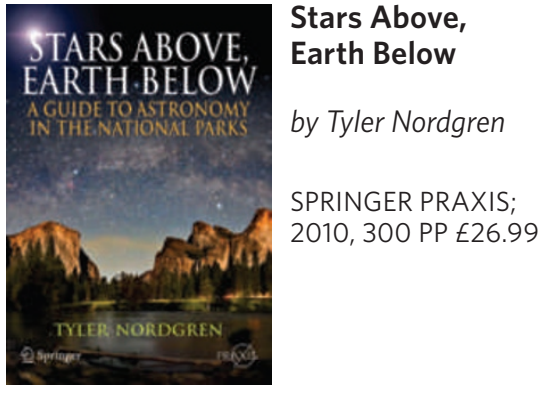

n Stars Above, Earth Below Tyler Nordgren talks not just about stars, but about virtually all heavenly objects. A substantial amount of space is devoted to our Solar System. Earth, especially the geological features found in the national parks of the United States, is successfully used to explain what we see in the heavens above. Much of the book praises the beauty of our world: geological features are wonderful to look at, and there is also beauty in understanding the processes that are acting above our heads and below our feet.

Nordgren approaches the subject from many different directions, but successfully ties them all together into a wonderful, poetic and coherent whole that fits the grandeur associated with the topic. From the start we are invited to view the subject of astronomy in a different way. He writes: "Come see the Milky Way. Come to the mountains, come to the forests, come to the national parks and see for yourself our Galaxy's power to inspire. Its glistening band across a jet black sky is the surest sight that we are part of a vast and complex Universe awaiting our discovery". The sky is described at different times of the year, as viewed from different US national parks. As expected, the book explains what can be seen. But along the way we get much background on how we came to understand what we see.

The first chapter, 'Come see the Milky Way', explores the history of humanity's thoughts about the heavens. For example, the Luiseño Native American tribe of the Los Angeles basin thought of the Milky Way as a net woven from milkweed fibres that was used to launch the Sun, after several attempts, into its proper motion through the sky. Nordgren paints a picture of the Milky Way as a landscape with bright regions that are closer and dim regions that are farther away. He describes an especially bright region near the constellation Sagittarius - the Lagoon Nebula — as a cloud of interstellar gas and dust. Such nebulae are the birthplaces of stars like our Sun, which form from the gravitational contraction of the gas and dust. The light from the bright young stars illuminates the surrounding dust, making it visible to us. The section is a fine blend of poetry, history and scientific understanding.

In a chapter about the Sun, Nordgren introduces the phases of the Moon, which are commonly understood. But then he continues onto the phases of Venus and Mercury, which few of us have seen. We learn how it is possible to predict exactly when and where the solar eclipse of 2017 will take place - and that the Grand Teton National Park, Wyoming, is one of the places where it can be observed. But the book takes us far beyond solar eclipses observed on Earth, showing images of an eclipse of one of the moons of Mars, as seen from the Mars rover Opportunity. In this case, the moon is not large enough to completely block out the Sun, as it does on Earth.

\section{The Luiseño Native American tribe of the Los Angeles basin thought of the Milky Way as a net woven from milkweed}

fibres that was used to launch the Sun, after several
attempts, into its proper
motion through the sky.

With the motion of the planets, Nordgren introduces the implications of gravity. The history of our understanding of planetary motion is introduced by way of Kepler and Newton. Kepler rose to fame in 1596, when he suggested that the spacing between the six known planets was set by God. He spent a decade working with the data of a contemporary astronomer, Tycho Brahe, to prove his hypothesis, but in the end the data did not fit his ideas. He then developed the three great 'Kepler laws' of planetary motion, which are still in use today. Nearly 60 years after Kepler's death, Newton postulated his law of gravity, which could explain the basis of Kepler's laws. The implications of Einstein's theory of relativity for predictions of planetary motion, and the ability of large masses to warp space and deflect light, are then discussed. Einstein's theory was able to account for previously inexplicable deviations in the orbit of Mercury from its elliptical path. Observations of the apparent positions of stars whose light passed very close to the Sun during a solar eclipse in 1919 later confirmed the theory.

Following the discussion on gravity, Nordgren moves onto the concept of tides. After explaining Earth's tides — which are understood by most physical scientists, but probably not by many laypeople - he talks about the effects of the tides on other objects in the Solar System. We see pictures of remarkable cracks and ridges on the surfaces of Jupiter's moon Europa, which otherwise has no topographical features such as mountains and valleys. The cracks are caused by variable gravitational pull on the surface of the moon as it travels in its elliptical orbit. For pairs of binary stars, the tides caused by one star can even affect the shape of the other star.

Each chapter ends with a 'See for yourself' section that is essentially a guided tour of some of the examples. In many cases these sections include charts that map both the sky at different times of the year and the objects of interest. Throughout the book examples from Earth and other planets are used to illustrate the processes that shape the Universe. For example, volcanism is discussed in the context of the Yellowstone and Hawai'i Volcanoes national parks. That radioactive heating is the energy source for volcanism on Earth is contrasted with the truly remarkable conditions on Jupiter's moon Io, where essentially continuous volcanism is powered by the tidal heating from Jupiter.

This book is written for the layperson who is interested in understanding the world in which we live, or in appreciating the beauty that comes with it. The explanations are very clear, with examples at a level any interested reader can understand. Yet the professional scholar working in geoscience, planetary science or astronomy will also find it an enlightening read. Even as a practising professional for many years in planetary sciences, I learnt a great deal from this book. And I particularly enjoyed the journey.

\section{REVIEWED BY WILLIAM BOYNTON}

William Boynton is in the Lunar and Planetary Laboratory, University of Arizona, Tucson, Arizona 85721, USA. 